\title{
The Exercise Science Toolkit: a web application for exercise and health science students
}

\section{Norton, Kevin ${ }^{a}$ and Norton, Lynda ${ }^{b}$}

${ }^{a}$ School of Health Sciences, University of South Australia, Australia, ${ }^{b}$ Discipline of Health and Exercise Sciences, Flinders University, Australia.

\begin{abstract}
The Exercise Science Toolkit is a web-based educational software program for exercise and health science tertiary students and professionals. The software was developed to facilitate student engagement with essential knowledge, skills and protocols in these disciplines, provide opportunities to conduct analyses on empirical data or simulations on an unlimited supply of virtual clients including difficult or rare cases, encourage overlearning opportunities at their own pace and in their own time, and shift the emphasis from passive knowledge delivery to creative acquisition and application of knowledge. The toolkit is evidence-based with numerous databases on ageand sex-specific population norms built from systematic searches. It uses established internationally accepted protocols for the analytical tools and test procedures. The toolkit has over 40 screens or tools to engage users in a variety of pedagogical modes including pre-class preparation, laboratories in real time, individual and group problem-based learning, online and distance education.
\end{abstract}

Keywords: analytic tool; body composition; fitness testing; health profiles; exercise physiology; virtual people 


\section{Introduction}

Online delivery and e-learning are growing rapidly as part of multimodal pedagogies, including disciplines such as health and clinical sciences (Ellman \& Schwartz, 2016). Acquisition and application of knowledge involves challenges in educating students and professionals in methodological issues and protocols, data analytics, knowledge integration, and communication skills. Our goal, therefore, was to develop a comprehensive online analytical and exploratory software program called the Exercise Science Toolkit (EST) to help students in exercise- and health-related disciplines develop proficiencies in these areas. The tools within the program cover key content including pre-exercise screening procedures, health risk factor assessment, physiological responses to exercise, performance testing across the three energy systems, body composition analysis and basic statistical/analytical calculators. Numerous approaches are used to reinforce knowledge and its context using population norms and variations, and novel 'what-if' functionality. Simulation strategies have also been created to supply an unlimited number of virtual clients offering unique learning opportunities with a vast array of individuals, and in a range of delivery modes. The URL for free access to the toolkit is: www.exercisesciencetoolkit.com (Norton \& Norton, 2018).

\section{Rationale for the Exercise Science Toolkit}

Understanding foundation elements of exercise, sport and health sciences courses takes time and rehearsal, especially practical skills that are applied as professionals. Students need frequent exposure to laboratory experiences to process the: (1) physiological/biochemical or performance variable being measured, (2) units of measurement, and (3) knowledge integration with contextual references such as what is 'normal', 'extreme', 'plausible', 'a risk factor', 'world class', 'low' for a specific type of athlete or diseased patient etc. Putting their own measures into perspective is almost invariably how students associate previous knowledge with new information to better understand the meaning, including the units of measurement (Mishra \& Koehler, 2006). For example, after collecting physiological or performance data for a variable students will often ask 'am I normal?' or 'what is normal?' or 'what is a good value?' or 'what does that value mean?' The EST allows students to engage with the tool and add contextual layers to measured data in an inquiry-based learning framework (Magnusson et al., 1999). Showing students how their own 'score' relates to other reference scores or benchmark populations helps them to assimilate information while reinforcing other elements such as probability and statistics.

The EST is a series of tools or calculators that compute results and integrates these with other information, for example, generating training zones based on the results of anaerobic 
threshold tests. It also allows comparisons with reference norms across a range of sports, age- and sex-groups, and other clinical sub-populations based on lifestyle behaviours. It uses data from published databases on population and sports-specific norms and is therefore credible and reliable. The laboratory results per se are important but mean relatively little without interpretation and exploring how they fit with reference values of populations (eg., male versus female; trained versus untrained; across various sports, with ageing and chronic health conditions, within the range of human capacities etc). This capacity encourages interactivity, particularly when combined with the 'what-if' functionality on each tool. The tools create opportunities to introduce explanations about why values are like they are and how results can be applied in sports and health science roles. The EST has been developed for multiple modes of delivery including flipped classes, face-to-face classroom activities and within online courses. It is designed to be visually appealing, modifiable and interactive. Moreover, international standards are adopted throughout such as in anthropometry assessment (Norton et al., 1996) and protocols for measuring physical power, aerobic fitness and anaerobic threshold testing (Tanner \& Gore 2013). Currently, six different national biometric datasets are embedded in the EST.

\section{Exercise Science Toolkit structure and functionality}

The EST has multiple analytical tools on approximately 40 screens. These are grouped within six modules covering (1) screening and risk factor assessment, (2) fitness testing across all energy systems, (3) body composition analysis, (4) analyzing blood biomarkers, (5) finding sports that match body size and shape, and (6) generating virtual profiles for population analytics. The EST has two 'layers', or ways of using the program: (1) for analysis of laboratory-obtained raw data that students might collect in class, or professionals in their practice, and (2) to create a virtual person (VP) to further investigate.

Each VP is created in real time and there are unlimited numbers of profiles that can be generated. This is because each VP is produced using realistic algorithms linking age, sex, and lifestyle behaviours such as physical activity habits, sedentary patterns and smoking, to outcome variables in areas including health risk factors, medical conditions, body composition and fitness parameters across the energy systems. Each VP profile generated results in $~ 200$ random normal variables created for physical, physiological, biochemical and behavioural characteristics that are based on published mathematical models of physiological responses to exercise and correlations between variables to guarantee plausible combinations. Large comparative databases were compiled following systematic reviews of normative data for fitness and physical attributes of elite athletes in over 100 sports, and results from national health surveys for age- and sex-comparisons. Generating a VP is particularly useful in situations where real data have not been collected, where access 
to testing equipment is not possible, or where it is problematic for students to gain access to clients that are difficult to practice on in real life (elite athletes, elderly, with chronic conditions or multiple health risk factors etc).

\section{Why simulate?}

In their professional lives exercise/health science specialists administer a wide variety of physiological tests to a broad range of clients using sophisticated and expensive equipment. Difficulties are encountered when exposing students to sub-populations, for example, to athletes in real life. Elite athletes are rare and are reluctant to interrupt training. Other subgroups, such as cardiac rehabilitation patients involve additional risks when testing and so are seldom entrusted to students without direct supervision. Simulation using virtual people overcomes these obstacles allowing students to create world champions or people with chronic illnesses and then explore relationships among lifestyle behaviours, demographic information, physiological and fitness parameters. They can explore these contexts multiple times and at their own pace, as well as offering extensive opportunities for application of knowledge and skills.

Simulation facilitates overlearning where students can gain a high volume of training in data interpretation and knowledge integration across the spectrum of human capabilities. Additionally, professionals in real life need to work with absolute confidence and certainty because serious errors are unacceptable. Simulation supports safe, accelerated testing and the EST maintains interest by constantly varying the human material.

The simulation strategy of the EST promotes problem-based learning. It has embedded capacity for trial-and-error approaches. For example, many tools have 'what-if' functionality where users can monitor the effect of changing input parameters on response variables such as health status, blood biomarkers, body composition and fitness characteristics. Simulation also brings with it open-ended analytical capabilities allowing free play to trial novel solutions and explore relationships among variables. The EST allows students to generate virtual populations (up to 5000 per iteration), export the database files and then investigate correlations and probability patterns among variables.

\section{Exercise Science Toolkit content}

An overview of some of the screens in the six modules is presented below. The opening screen in figure 1 shows there is an initial option for either empirical data entry or to generate a VP profile. To narrow the sub-population generated, users can select specific age, sex or physical activity groups. 


\subsection{Screening and Risk Factor Assessment module}

Pre-exercise screening is part of the duty of care for those involved in exercise prescription. The screening module in the EST is based on the Australian model (Norton \& Norton, 2011) and can be used for real clients to assist in managing medical conditions and in constructing appropriate programs for people beginning exercise. It also allows students to investigate a range of VP that would otherwise be rare for students to see, for example, the elderly, and those in poorer health and fitness states etc, but yet graduates may be expected to handle immediately upon graduation. This facilitates decision-making and crosschecking of these decisions in the safety of dealing with a VP rather than real clients. Comprehensive 'what-if' functionality is embedded in the module to facilitate exploration of health risk factors, absolute risk of cardiovascular disease and life expectancy as behaviours and biomarkers are altered.
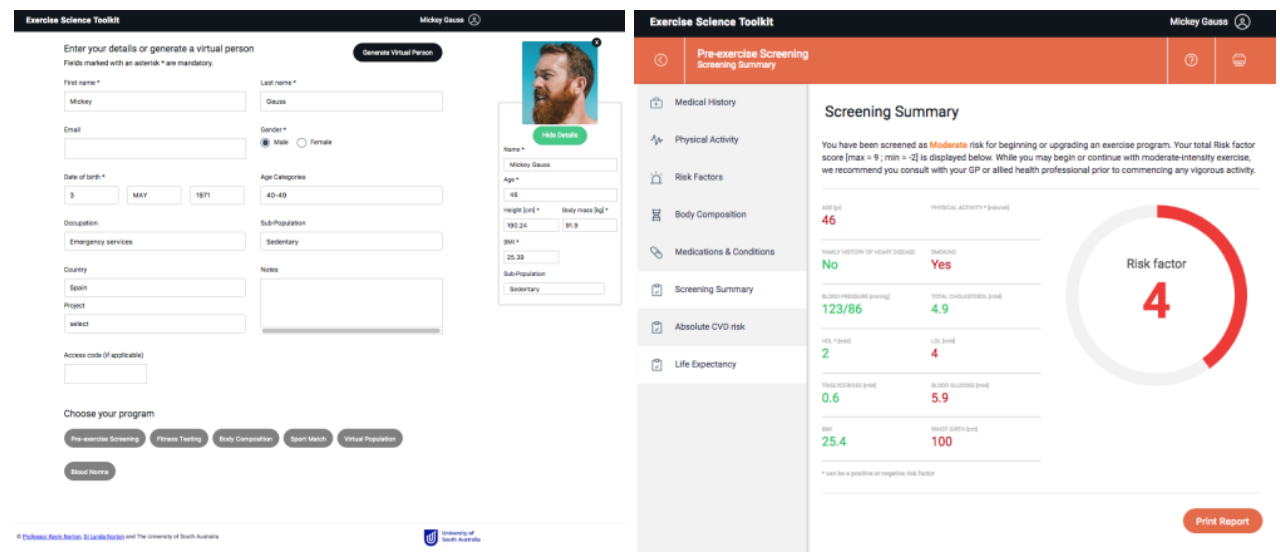

Figure 1. The opening screen in the EST showing a generated virtual person (sedentary male, 46 y, BMI 25.4)(left); The Pre-exercise Screening and Risk Factor Assessment module summary screen (right).

\subsection{Fitness Testing module}

The Fitness testing module is structured to reflect a range of tests associated with each of the energy systems. On most of these screens there are both population-based norms (5year age- and sex-specific groups) as well as a contemporary database of elite athletes reported in the scientific literature. There are printout options for each screen and numerous graphing and comparison functions. 

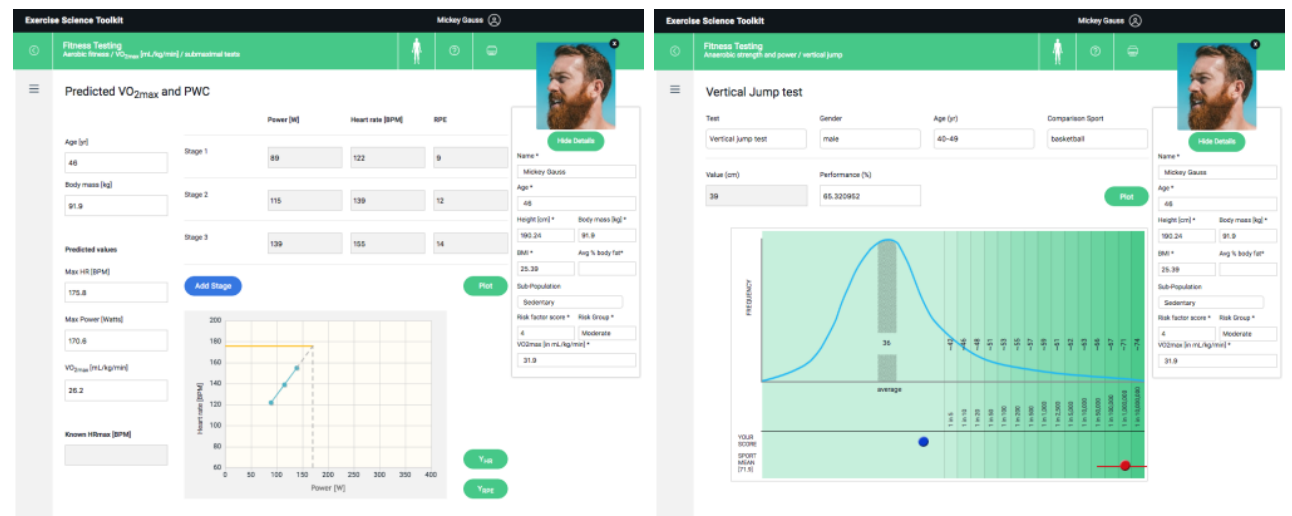

Figure 2. Example screens for the Fitness Testing module; (left) aerobic fitness test results including heart rate and workloads for a submaximal exercise test to predict maximal oxygen uptake, and (right) probability plot for the vertical jump test results and an elite sport comparison (elite basketballers).

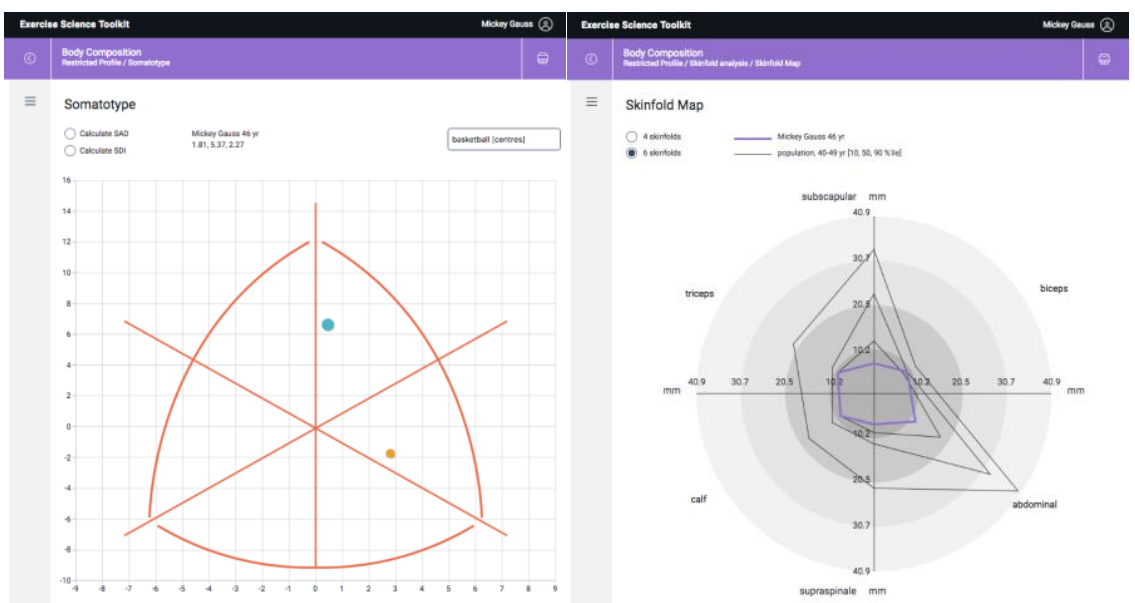

Figure 3. Example Body Composition module screens for the VP. The left panel shows the calculated somatotype of the VP and a comparison plot for elite-level basketabllers. The right panel shows a skinfold map for 6 skinfolds relative to age- and sex-specific population norms.

\subsection{Body Composition and Sport Match modules}

The Body Composition module allows the user to input anthropometry data and then explore outputs such as: comparisons against population norms, \% body fat prediction, skinfold plots, somatotype, fractionation of body mass, and various types of reliability analyses for students to check their skill competencies. The Sport Match module uses the inputs of a range of body measurements (size, shape and composition) and a bivariate probability function to determine the profile's best fit among about 100 sports (Norton \& 
Olds, 2001). The normative data are from published information of the highest quality athletes available and the degree of 'best fit' is represented by an 'overlap score' out of 100 . Students can perform 'what-if' scenarios to modify inputs and explore the sports rankings thereby linking body proportions and composition with sport functionality and athletic performance (biomechanics, fitness etc).

\subsection{Blood Biomarker module}

Users can enter data to determine percentile ranks for up to nine biomarkers and to reinforce units of measurement and risk factor threshold levels for key health indicators. Databases were constructed from published national health surveys and large epidemiological studies across six countries and cover ages 18-75 yr.

\subsection{Virtual Population module}

To facilitate investigation among the $200 \mathrm{VP}$ variables a 'Virtual population' function has been developed. This allows thousands of VP profiles to be generated and explored for patterns such as correlations or ageing trends, differences across sexes or, for example, when analysing different disease conditions, sedentariness and activity levels.
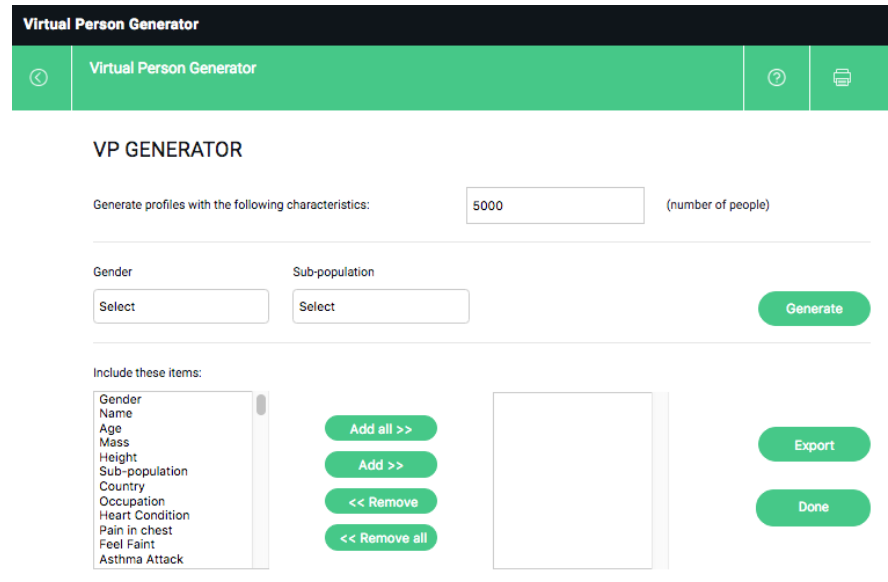

Figure 4. The Virtual Population module can generate up to 5000 VP profiles per iteration. Specific subpopulations can be chosen and laboratory exercise tailored for particular curriculum focus areas.

\section{Conclusion}

The EST has been developed to (1) reinforce applications of correct measurement units and ranges, (2) understand the breadth of human capabilities and limitations, (3) explore 
relationships among behaviours, health and fitness for both individuals and in populations, (4) calculate, compare, integrate, graph and print data, either real or virtual, (5) conduct interactive analyses on health and fitness parameters, including unusual and difficult cases to experience in real life, (6) instruct protocols and laboratory procedures, and (7) apply simple statistical methods to calculate reliability of skills and methods.

The EST facilitates dynamic, interactive learning sessions where students can use empirical or simulated data in a variety of pedagogical contexts such as problem-based learning, team-based learning, 'what-if' scenarios, clinical simulation exercises, and patient decisionmaking. Teachers can also develop and share laboratory ideas and supporting resources such as quizzes, video clips and project designs that utlize the EST functionality.

\section{References}

Ellman, M.S., \& Schwartz, M.L. (2016). Online Learning Tools as Supplements for Basic and Clinical Science Education. Journal of Medical Education and Curricular Development, 3 109-114 doi:10.4137/JMECD.S18933.

Magnusson, S, Krajcik, J. \& Borko, H. (1999). Examining Pedagogical Content Knowledge, Chapter 4 in Volume 6 of the series Science \& Technology Education Library pp 95-132. Nature, Sources, and Development of Pedagogical Content Knowledge for Science Teaching.

Mishra, P. \& Koehler, M. (2006). Technological Pedagogical Content Knowledge: A Framework for Teacher Knowledge. Teachers College Record Volume 108 Number 6, p. 1017-1054. http://www.tcrecord.org ID Number: 12516, Date Accessed: 28/01/2018.

Norton, K. \& Norton, L. (2011). Pre-exercise screening. Guide to the Australian adult preexercise screening system. Published jointly by Exercise \& Sports Science Australia, Fitness Australia and Sports Medicine Australia.

Norton, K. \& Norton, L. (2018). Exercise Science Toolkit [Computer software]. Retrieved from http://exercisesciencetoolkit.com.

Norton, K. \& Olds, T. (2001). Morphological evolution of athletes over the 20th century: causes and consequences. Sports Medicine, 31, 763-783.

Norton, K.I., Whittingham, N., Carter, J.E.L., Kerr, D., Gore, C.J. \& Marfell-Jones, M. (1996). Measurement techniques in anthropometry. In K. Norton and T.S. Olds (Eds.) Anthropometrica, 25 - 75, Sydney: UNSW Press.

Tanner, R.K, \& Gore, C.J. (2013). Physiological tests for elite athletes $2^{\text {nd }}$ ed. Illinois: Human Kinetics. 